\title{
HEAT KERNEL BOUNDS FOR ELLIPTIC PARTIAL DIFFERENTIAL OPERATORS IN DIVERGENCE FORM WITH ROBIN-TYPE BOUNDARY CONDITIONS II
}

\author{
FRITZ GESZTESY, MARIUS MITREA, ROGER NICHOLS, AND EL MAATI OUHABAZ
}

(Communicated by Joachim Krieger)

Dedicated with great pleasure to E. Brian Davies on the occasion of his 70th birthday.

Abstract. The principal aim of this short note is to extend a recent result on Gaussian heat kernel bounds for self-adjoint $L^{2}\left(\Omega ; d^{n} x\right)$-realizations, $n \in \mathbb{N}, n \geqslant 2$, of divergence form elliptic partial differential expressions $L$ with (nonlocal) Robin-type boundary conditions in bounded Lipschitz domains $\Omega \subset \mathbb{R}^{n}$, where

$$
L u=-\sum_{j, k=1}^{n} \partial_{j} a_{j, k} \partial_{k} u .
$$

The (nonlocal) Robin-type boundary conditions are then of the form

$$
\nu \cdot A \nabla u+\Theta\left[\left.u\right|_{\partial \Omega}\right]=0 \text { on } \partial \Omega,
$$

where $\Theta$ represents an appropriate operator acting on Sobolev spaces associated with the boundary $\partial \Omega$ of $\Omega$, and $\nu$ denotes the outward pointing normal unit vector on $\partial \Omega$.

\section{INTRODUCTION}

This note represents an addendum to the recent paper [4 devoted to a new class of self-adjoint realizations $L_{\theta, \Omega}$ in $L^{2}\left(\Omega ; d^{n} x\right)$ of elliptic partial differential expressions in divergence form,

$$
L=-\sum_{j, k=1}^{n} \partial_{j} a_{j, k} \partial_{k},
$$

on bounded Lipschitz domains $\Omega \subset \mathbb{R}^{n}, n \geqslant 2$, with Robin boundary conditions of the form $\nu \cdot A \nabla u+\theta\left(\left.u\right|_{\partial \Omega}\right)=0$. (Here $\nu$ denotes the outward pointing normal unit vector and $\theta$ is a suitable function on the boundary $\partial \Omega$ of $\Omega$.) Following [3], we put particular emphasis in [4] on developing a theory of nonlocal Robin boundary conditions where the function $\theta$ on $\partial \Omega$ is replaced by a suitable operator

Received by the editors August 22, 2013.

2010 Mathematics Subject Classification. Primary 35J15, 35J25, 47D06; Secondary 46E35, 47A10, 47D07.

Key words and phrases. Positivity preserving semigroups, elliptic partial differential operators, Robin boundary conditions, heat kernel bounds, Green's function bounds.

The work of the second author was partially supported by the Simons Foundation Grant \# 281566 and a University of Missouri Research Leave Grant.

The third author gratefully acknowledges support from an AMS-Simons Travel Grant.

The work of the fourth author was partly supported by the ANR project "Harmonic Analysis at its Boundaries," ANR-12-BS01-0013-02. 
$\Theta$ acting in $L^{2}\left(\partial \Omega ; d^{n-1} \omega\right)$, with $d^{n-1} \omega$ representing the surface measure on $\partial \Omega$. (More precisely, $\Theta$ acts in appropriate Sobolev spaces on the boundary of $\Omega$ ). The resulting self-adjoint operator in $L^{2}\left(\Omega ; d^{n} x\right)$ is then denoted by $L_{\Theta, \Omega}$ and we study its resolvent and semigroup, proving a Gaussian heat kernel bound and a bound for the Green's function of $L_{\Theta, \Omega}$.

To keep this note short, we will refer the reader to the detailed paper [4. Especially, we refer to the extensive introduction and long list of references contained therein. In particular, we will only reproduce that material from 4 that is absolutely necessary to read this note.

In Section 3 we provide a bit of background and restate the principal result of [4] and then our current improvement based on a natural, additional condition. Section 4 then provides some concrete illustrations.

Finally, we briefly summarize some of the notation used in this paper: Let $\mathcal{H}$ be a separable complex Hilbert space, $(\cdot, \cdot)_{\mathcal{H}}$ the scalar product in $\mathcal{H}$ (linear in the second argument), and $I_{\mathcal{H}}$ the identity operator in $\mathcal{H}$.

Next, if $T$ is a linear operator mapping (a subspace of) a Hilbert space into another, then $\operatorname{dom}(T)$ and $\operatorname{ker}(T)$ denote the domain and kernel (i.e., null space) of $T$. The closure of a closable operator $S$ is denoted by $\bar{S}$. The spectrum, essential spectrum, discrete spectrum, and resolvent set of a closed linear operator in a Hilbert space will be denoted by $\sigma(\cdot), \sigma_{\text {ess }}(\cdot), \sigma_{\mathrm{d}}(\cdot)$, and $\rho(\cdot)$, respectively.

The Banach spaces of bounded and compact linear operators on a separable complex Hilbert space $\mathcal{H}$ are denoted by $\mathcal{B}(\mathcal{H})$ and $\mathcal{B}_{\infty}(\mathcal{H})$, respectively. The analogous notation $\mathcal{B}\left(\mathcal{X}_{1}, \mathcal{X}_{2}\right), \mathcal{B}_{\infty}\left(\mathcal{X}_{1}, \mathcal{X}_{2}\right)$ will be used for bounded and compact operators between two Banach spaces $\mathcal{X}_{1}$ and $\mathcal{X}_{2}$.

Given a $\sigma$-finite measure space, $(M, \mathcal{M}, \mu)$, the product measure on $M \times M$ will be denoted by $\mu \otimes \mu$. Without loss of generality, we also denote the completion of the product measure space $(M \times M, \mathcal{M} \otimes \mathcal{M}, \mu \otimes \mu)$ by the same symbol and always work with this completion in the following.

For $a, b \in \mathbb{C}^{n}$, we use the Euclidean pairing $\langle a, b\rangle_{\mathbb{C}^{n}}=\sum_{j} a_{j} b_{j}=(\bar{a}, b)_{\mathbb{C}^{n}}$.

\section{NONLOCAL ROBIn BOUNDARY CONDITIONS}

We start by recalling our basic notation on positivity preserving/improving operators.

Hypothesis 2.1. Let $(M, \mathcal{M}, \mu)$ denote a $\sigma$-finite, separable measure space associated with a nontrivial positive measure (i.e., $0<\mu(M) \leqslant \infty$ ).

The set of nonnegative elements $0 \leqslant f \in L^{2}(M ; d \mu)$ (i.e., $f(x) \geqslant 0 \mu$-a.e.) is a cone in $L^{2}(M ; d \mu)$, closed in the norm and weak topologies.

Definition 2.2. Let $A$ be a bounded linear operator in $L^{2}(M ; d \mu)$. Then $A$ is called positivity preserving (resp., positivity improving) if

$$
0 \neq f \in L^{2}(M ; d \mu), f \geqslant 0 \text { implies } A f \geqslant 0 \text { (resp., } A f>0 \text { ). }
$$

Given two bounded operators $A$ and $B$ on $L^{2}(M ; d \mu)$ such that $A$ is positivity preserving, we say that $B$ is dominated by $A$ if

$$
|B f| \leqslant A|f|, \quad f \in L^{2}(M ; d \mu) .
$$

Here and in the rest of this paper, all the inequalities (and equalities) are understood in the $\mu$-a.e. sense. 
Turning our attention to integral operators in $L^{2}(M ; d \mu)$ with associated integral kernels $A(\cdot, \cdot)$ on $M \times M$, we assume that

$$
A(\cdot, \cdot): M \times M \rightarrow \mathbb{C} \text { is } \mu \otimes \mu \text {-measurable, }
$$

and introduce the integral operator $A$ associated with the integral kernel $A(\cdot, \cdot)$ as follows:

$$
(A f)(x):=\int_{M} A(x, y) f(y) d \mu(y) \text { for } \mu \text {-a.e. } x \in M, f \in L^{2}(M ; d \mu) .
$$

This means that $A(x, \cdot) f(\cdot)$ is absolutely integrable over $M$ for $\mu$-a.e. $x \in M$ and $\int_{M} A(\cdot, y) f(y) d \mu(y) \in L^{2}(M ; d \mu)$.

Suppose that $A$ is bounded on $L^{2}(M ; d \mu)$. Then it is a classical fact that $A$ is positivity preserving if and only if

$$
A(\cdot, \cdot) \geqslant 0 \mu \otimes \mu \text {-a.e. on } M \times M .
$$

Similarly, if $B(x, y)$ denotes the integral kernel of an integral operator $B$ that is bounded on $L^{2}(M ; d \mu)$, then $B$ is dominated by $A$ if and only if

$$
|B(\cdot, \cdot)| \leqslant A(\cdot, \cdot) \mu \otimes \mu \text {-a.e. on } M \times M \text {. }
$$

Next we briefly turn to the basics for divergence form elliptic partial differential operators with (nonlocal) Robin-type boundary conditions in $n$-dimensional, bounded, Lipschitz domains, corresponding to differential expressions $L$ given by

$$
L u:=-\sum_{j, k=1}^{n} \partial_{j} a_{j, k} \partial_{k} u .
$$

For basic facts on Sobolev spaces on $\Omega$ or $\partial \Omega$ and Dirichlet and Neumann trace operators, as well as the choice of notation used below, we refer to [4, Appendix A]. For the basics on sesquilinear forms and operators associated with them we refer to [2, 6] and [4, Appendix B].

In the remainder of this section we make the following assumption:

Hypothesis 2.3. Let $n \in \mathbb{N}, n \geqslant 2$.

(i) Assume that $\Omega \subset \mathbb{R}^{n}$ is a bounded Lipschitz domain.

(ii) Suppose that the matrix

$$
A(\cdot)=\left(a_{j, k}(\cdot)\right)_{1 \leqslant j, k \leqslant n}
$$

satisfies $A \in L^{\infty}\left(\Omega ; d^{n} x\right)^{n \times n}$ and is real symmetric a.e. on $\Omega$. In addition, given $0<a_{0}<a_{1}<\infty$, assume that $A$ satisfies the uniform ellipticity conditions

$$
a_{0} I_{n} \leqslant A(x) \leqslant a_{1} I_{n} \text { for a.e. } x \in \Omega .
$$

Above, $I_{n}$ represents the identity matrix in $\mathbb{C}^{n}$ and we will denote the identity operators in $L^{2}\left(\Omega ; d^{n} x\right)$ and $L^{2}\left(\partial \Omega ; d^{n-1} \omega\right)$ by $I_{\Omega}$ and $I_{\partial \Omega}$, respectively. Also, in the sequel, the sesquilinear form

$$
\langle\cdot, \cdot\rangle_{s}=H_{H^{s}(\partial \Omega)}\langle\cdot, \cdot\rangle_{H^{-s}(\partial \Omega)}: H^{s}(\partial \Omega) \times H^{-s}(\partial \Omega) \rightarrow \mathbb{C}, \quad s \in[0,1],
$$

(antilinear in the first, linear in the second factor), will denote the duality pairing between $H^{s}(\partial \Omega)$ and

$$
H^{-s}(\partial \Omega)=\left(H^{s}(\partial \Omega)\right)^{*}, \quad s \in[0,1],
$$


such that

$$
\begin{aligned}
& \langle f, g\rangle_{s}=\int_{\partial \Omega} d^{n-1} \omega(\xi) \overline{f(\xi)} g(\xi), \\
& \text { whenever } f, g \in L^{2}\left(\partial \Omega ; d^{n-1} \omega\right),
\end{aligned}
$$

where $d^{n-1} \omega$ stands for the surface measure on $\partial \Omega$.

One observes that the inclusion

$$
\iota: H^{s_{0}}(\Omega) \hookrightarrow\left(H^{r}(\Omega)\right)^{*}, \quad s_{0}>-1 / 2, r>1 / 2,
$$

is well-defined and bounded.

Next, we wish to describe a weak version of the normal trace operator associated with $L$ in (2.7), considered in a bounded Lipschitz domain. To set the stage, assume Hypothesis 2.3 and introduce the weak Neumann trace operator

$$
\widetilde{\gamma}_{N}:\left\{u \in H^{s+1 / 2}(\Omega) \mid L u \in H^{s_{0}}(\Omega)\right\} \rightarrow H^{s-1}(\partial \Omega), \quad s_{0}>-1 / 2,
$$

as follows: Given $u \in H^{s+1 / 2}(\Omega)$ with $L u \in H^{s_{0}}(\Omega)$ for some $s_{0}>-1 / 2$ and $s \in(0,1)$, we set (with $\iota$ as in (2.13) for $r:=3 / 2-s>1 / 2$ )

$$
\begin{aligned}
\left\langle\phi, \widetilde{\gamma}_{N} u\right\rangle_{1-s}:= & H^{1 / 2-s}(\Omega)^{n} \\
& -{ }_{H^{3 / 2-s}(\Omega)}\langle\Phi, \iota(L u)\rangle_{\left(H^{3 / 2-s}(\Omega)\right)^{*}},
\end{aligned}
$$

for all $\phi \in H^{1-s}(\partial \Omega)$ and $\Phi \in H^{3 / 2-s}(\Omega)$ such that $\gamma_{D} \Phi=\phi$, where we denoted the Dirichlet trace operator by $\gamma_{D}$. We recall that

$$
\widetilde{\gamma}_{N}:\left\{u \in H^{1}(\Omega) \mid L u \in H^{s_{0}}(\Omega)\right\} \rightarrow H^{-1 / 2}(\partial \Omega), \quad s_{0}>-1 / 2,
$$

is well-defined, linear, and bounded.

Moving on, we take up the task of describing the precise conditions that we impose on the nonlocal Robin boundary operator $\Theta$.

Hypothesis 2.4. Assume Hypothesis 2.3, suppose that $\delta>0$ is a given number, and assume that $\Theta \in \mathcal{B}\left(H^{1 / 2}(\partial \Omega), H^{-1 / 2}(\partial \Omega)\right)$ is a self-adjoint operator which can be written as

$$
\Theta=\Theta^{(1)}+\Theta^{(2)}+\Theta^{(3)},
$$

where $\Theta^{(j)}, j=1,2,3$, have the following properties: There exists a closed sesquilinear form $\mathfrak{q}_{\partial \Omega}^{(0)}$ in $L^{2}\left(\partial \Omega ; d^{n-1} \omega\right)$, with domain $H^{1 / 2}(\partial \Omega) \times H^{1 / 2}(\partial \Omega)$, which is bounded from below by $c_{\partial \Omega} \in \mathbb{R}$ such that if $\Theta_{\partial \Omega}^{(0)} \geqslant c_{\partial \Omega} I_{\partial \Omega}$ denotes the self-adjoint operator in $L^{2}\left(\partial \Omega ; d^{n-1} \omega\right)$ uniquely associated with $\mathfrak{q}_{\partial \Omega}^{(0)}$, then $\Theta^{(1)}=\widetilde{\Theta}_{\partial \Omega}^{(0)}$, the extension of $\Theta_{\partial \Omega}^{(0)}$ to an operator in $\mathcal{B}\left(H^{1 / 2}(\partial \Omega), H^{-1 / 2}(\partial \Omega)\right)$. In addition,

$$
\Theta^{(2)} \in \mathcal{B}_{\infty}\left(H^{1 / 2}(\partial \Omega), H^{-1 / 2}(\partial \Omega)\right),
$$

whereas $\Theta^{(3)} \in \mathcal{B}\left(H^{1 / 2}(\partial \Omega), H^{-1 / 2}(\partial \Omega)\right)$ satisfies

$$
\left\|\Theta^{(3)}\right\|_{\mathcal{B}\left(H^{1 / 2}(\partial \Omega), H^{-1 / 2}(\partial \Omega)\right)}<\delta .
$$

The self-adjoint realization of the differential expression (2.7) equipped with nonlocal Robin-type boundary conditions associated with an operator $\Theta$ as above is recorded below. 
Theorem 2.5 (4]). Assume Hypothesis 2.4, where the number $\delta>0$ is taken to be sufficiently small relative to the Lipschitz character of $\Omega$; more precisely, suppose that $0<\delta \leqslant \frac{1}{6}\left\|\gamma_{D}\right\|_{\mathcal{B}\left(H^{1}(\Omega), H^{1 / 2}(\partial \Omega)\right)}^{-2}$. In addition, consider the sesquilinear form $\mathfrak{Q}_{\Theta, \Omega}(\cdot, \cdot)$ defined on $H^{1}(\Omega) \times H^{1}(\Omega)$ by

$$
\begin{array}{r}
\mathfrak{Q}_{\Theta, \Omega}(u, v):=\int_{\Omega} d^{n} x\langle\overline{(\nabla u)(x)}, A(x)(\nabla v)(x)\rangle_{\mathbb{C}^{n}}+\left\langle\gamma_{D} u, \Theta \gamma_{D} v\right\rangle_{1 / 2}, \\
u, v \in H^{1}(\Omega) .
\end{array}
$$

Then the form $\mathfrak{Q}_{\Theta, \Omega}(\cdot, \cdot)$ in (2.20) is symmetric, $H^{1}(\Omega)$-bounded, bounded from below, and closed in $L^{2}\left(\Omega ; d^{n} x\right)$. The self-adjoint operator $L_{\Theta, \Omega}$ uniquely associated with $\mathfrak{Q}_{\Theta, \Omega}$ on $L^{2}\left(\Omega ; d^{n} x\right)$ is then given by

$$
\begin{aligned}
& L_{\Theta, \Omega}=L, \\
& \operatorname{dom}\left(L_{\Theta, \Omega}\right)=\left\{u \in H^{1}(\Omega) \mid L u \in L^{2}\left(\Omega ; d^{n} x\right),\left(\widetilde{\gamma}_{N}+\Theta \gamma_{D}\right) u=0 \text { in } H^{-1 / 2}(\partial \Omega)\right\},
\end{aligned}
$$

and is self-adjoint and bounded from below on $L^{2}\left(\Omega ; d^{n} x\right)$. Moreover,

$$
\operatorname{dom}\left(\left|L_{\Theta, \Omega}\right|^{1 / 2}\right)=H^{1}(\Omega),
$$

and $L_{\Theta, \Omega}$, has purely discrete spectrum bounded from below. In particular,

$$
\sigma_{\mathrm{ess}}\left(L_{\Theta, \Omega}\right)=\emptyset \text {. }
$$

In the special case of Neumann boundary conditions (corresponding to $\Theta=0$ ), we use the notation

$$
\mathfrak{Q}_{N, \Omega}(\cdot, \cdot)=\mathfrak{Q}_{0, \Omega}(\cdot, \cdot), \quad L_{N, \Omega}=L_{0, \Omega} .
$$

Next, we briefly comment on the usual case of a local Robin boundary condition, that is, the scenario when $\Theta$ is the operator $M_{\theta}$, of pointwise multiplication by a real-valued function $\theta$ defined on $\partial \Omega$ :

Lemma 2.6 ([3]). Assume Hypothesis 2.3 and suppose that $\Theta=M_{\theta}$, the operator of pointwise multiplication by a real-valued function $\theta \in L^{p}\left(\partial \Omega ; d^{n-1} \omega\right)$, where

$$
p=n-1 \text { if } n>2 \text {, and } p \in(1, \infty] \text { if } n=2 .
$$

Then

$$
\Theta \in \mathcal{B}_{\infty}\left(H^{1 / 2}(\partial \Omega), H^{-1 / 2}(\partial \Omega)\right)
$$

is a self-adjoint operator which satisfies

$$
\|\Theta\|_{\mathcal{B}\left(H^{1 / 2}(\partial \Omega), H^{-1 / 2}(\partial \Omega)\right)} \leqslant C\|\theta\|_{L^{p}\left(\partial \Omega ; d^{n-1} \omega\right)}
$$

for some finite constant $C=C(\Omega, n, p) \geqslant 0$. In particular, the present situation $\Theta=M_{\theta}$ subordinates to the case $\Theta^{(2)}$ described in (2.18).

The $L^{2}$-realization of $L$ equipped with a Dirichlet boundary condition, $L_{D, \Omega}$, in $L^{2}\left(\Omega ; d^{n} x\right)$ formally corresponds to $\Theta=\infty$. Note that

$$
\begin{aligned}
& L_{D, \Omega}=L, \\
& \begin{aligned}
\operatorname{dom}\left(L_{D, \Omega}\right) & =\left\{u \in H^{1}(\Omega) \mid L u \in L^{2}\left(\Omega ; d^{n} x\right), \gamma_{D} u=0 \text { in } H^{1 / 2}(\partial \Omega)\right\} \\
& =\left\{u \in H_{0}^{1}(\Omega) \mid L u \in L^{2}\left(\Omega ; d^{n} x\right)\right\} .
\end{aligned}
\end{aligned}
$$


The well-known Beurling-Deny criteria (cf. [2], 6]) allow to prove positivity preserving for the semigroup (and, equivalently, the resolvent) of $L_{\Theta, \Omega}$. In order to achieve this, one assumes that

$$
\left\langle\gamma_{D}|u|, \Theta \gamma_{D}|u|\right\rangle_{1 / 2} \leqslant\left\langle\gamma_{D} u, \Theta \gamma_{D} u\right\rangle_{1 / 2}, \quad u \in H^{1}(\Omega)
$$

Under this assumption, one has for $u \in H^{1}(\Omega)$,

$$
\mathfrak{Q}_{\Theta, \Omega}(|u|,|u|) \leqslant \mathfrak{Q}_{\Theta, \Omega}(u, u),
$$

which by the first Beurling-Deny criterion is equivalent to positivity preserving of $e^{-t L_{\Theta, \Omega}}$. It is well-known that positivity preserving is valid for $e^{-t L_{D, \Omega}}$ and $e^{-t L_{N, \Omega}}$.

\section{Gaussian BOUndS}

Retaining Hypothesis 2.3 throughout this section, we now continue the discussion on divergence form elliptic partial differential operators $L_{\Theta, \Omega}$ with nonlocal Robin boundary conditions and focus on (Gaussian) heat kernel and Green's function bounds for $L_{\Theta, \Omega}$.

We will use the following heat kernel notation (for $t>0$, a.e. $x, y \in \Omega$ )

$$
\begin{aligned}
& K_{\Theta, \Omega}(t, x, y)=e^{-t L_{\Theta, \Omega}}(x, y), \quad K_{N, \Omega}(t, x, y)=e^{-t L_{N, \Omega}}(x, y), \\
& K_{D, \Omega}(t, x, y)=e^{-t L_{D, \Omega}}(x, y),
\end{aligned}
$$

and similarly for Green's functions (for $z \in \mathbb{C} \backslash \mathbb{R}$, a.e. $x, y \in \Omega$ ),

$$
G_{\Theta, \Omega}(z, x, y)=\left(L_{\Theta, \Omega}-z I_{\Omega}\right)^{-1}(x, y), \quad G_{N, \Omega}(z, x, y)=\left(L_{N, \Omega}-z I_{\Omega}\right)^{-1}(x, y),
$$

$$
G_{D, \Omega}(z, x, y)=\left(L_{D, \Omega}-z I_{\Omega}\right)^{-1}(x, y), \quad x \neq y .
$$

We recall that for $v \in L^{2}\left(\Omega ; d^{n} x\right), \bar{v}$ denotes the complex conjugate of $v$, and for two functions $u$ and $v$, the symbol $u . \bar{v} \geqslant 0$ means that the product of the functions, $u \bar{v}$, is nonnegative a.e. on $\Omega$.

To facilitate a comparison of our new Theorem 3.6 below with the principal result obtained in [4, we briefly recall the latter (in a slightly simplified form):

Theorem $3.1\left([4)\right.$. Assume Hypothesis 2.3 , suppose that $\Theta_{j}, j=1,2$, satisfy the assumptions introduced in Hypothesis 2.4, and denote by $L_{\Theta_{j}, \Omega}$ the operators in (2.21) uniquely associated with the sesquilinear forms $\mathfrak{Q}_{\Theta_{j}, \Omega}(\cdot, \cdot), j=1,2$, defined on $H^{1}(\Omega) \times H^{1}(\Omega)$ according to (2.20). Suppose, in addition, that

$$
\left\langle\gamma_{D}|u|, \Theta_{j} \gamma_{D}|u|\right\rangle_{1 / 2} \leqslant\left\langle\gamma_{D} u, \Theta_{j} \gamma_{D} u\right\rangle_{1 / 2}, \quad u \in H^{1}(\Omega), j=1,2 .
$$

Then, assuming $0 \leqslant \Theta_{1} \leqslant \Theta_{2}$, one has the positivity preserving relations

$0 \leqslant e^{-t L_{D, \Omega}}|f| \leqslant e^{-t L_{\Theta_{2}, \Omega}}|f| \leqslant e^{-t L_{\Theta_{1}, \Omega}}|f| \leqslant e^{-t L_{N, \Omega}}|f|, \quad f \in L^{2}\left(\Omega ; d^{n} x\right), t \geqslant 0$.

In addition, all semigroups appearing in (3.4) lie in the trace class,

$$
e^{-t L_{D, \Omega}}, e^{-t L_{\Theta_{j}, \Omega}}, e^{-t L_{N, \Omega}} \in \mathcal{B}_{1}\left(L^{2}\left(\Omega ; d^{n} x\right)\right), \quad j=1,2, t>0 .
$$

In particular, one has the Gaussian heat kernel bounds (for $t>0$, a.e. $x, y \in \Omega$ ),

$$
\begin{aligned}
0 & \leqslant K_{D, \Omega}(t, x, y) \leqslant K_{\Theta_{2}, \Omega}(t, x, y) \leqslant K_{\Theta_{1}, \Omega}(t, x, y) \leqslant K_{N, \Omega}(t, x, y) \\
& \leqslant C_{\alpha, a_{0}, \Omega} \max \left(t^{-n / 2}, 1\right) \exp \left\{-|x-y|^{2} /\left[4(1+\gamma) a_{1} t\right]\right\}, \quad \gamma \in(0,1) .
\end{aligned}
$$


To state the new results of this section we need a few preparations:

Let $\mathfrak{a}$ and $\mathfrak{b}$ be two sesquilinear, accretive, and closed forms on $H=L^{2}(M ; d \mu)$, and denote by $e^{-t A}$ and $e^{-t B}$ their associated semigroups, respectively.

Theorem 3.2. Suppose that the semigroup $e^{-t B}$ is positivity preserving and that $\operatorname{dom}(\mathfrak{a})=\operatorname{dom}(\mathfrak{b})$. Then the following assertions are equivalent.

(i) $\left|e^{-t A} f\right| \leqslant e^{-t B}|f|, f \in L^{2}(M ; d \mu), t \geqslant 0$.

(ii) $\operatorname{Re}(\mathfrak{a}(u, v)) \geqslant \mathfrak{b}(|u|,|v|)$ for all $u, v \in \operatorname{dom}(\mathfrak{a})$ such that $u . \bar{v} \geqslant 0$.

If both semigroups $e^{-t A}$ and $e^{-t B}$ are positivity preserving, then assertion $(i)$ is equivalent to

(iii) $\operatorname{Re}(\mathfrak{a}(u, v)) \geqslant \mathfrak{b}(u, v)$ for all nonnegative $u, v \in \operatorname{dom}(\mathfrak{a})$.

Sketch of proof. Since the semigroup $e^{-t B}$ is positivity preserving it follows from [6. Proposition 2.20] that $\operatorname{dom}(\mathfrak{a})$ is an ideal of itself and hence an ideal of $\operatorname{dom}(\mathfrak{b})$ because $\operatorname{dom}(\mathfrak{a})=\operatorname{dom}(\mathfrak{b})$ by hypothesis. We refer to [5] and [6, Definition 2.19] for the notion of an ideal in this context. Thus, the equivalence of items $(i)$ and $(i i)$ follows from [5, Corollary 3.4] (see also [6, Theorem 2.21]). If both semigroups are positivity preserving, the equivalence of items $(i)$ and $(i i i)$ follows from [5. Theorem 3.7] (see also [6, Theorem 2.24]).

Other criteria for the domination property in terms of forms in assertion $(i)$ for the case where $\operatorname{dom}(\mathfrak{a}) \neq \operatorname{dom}(\mathfrak{b})$ are given in [5] and [6, Ch. 2]. The equivalence of items $(i)$ and $($ iii) is also proved in [4].

We have the following result:

Theorem 3.3. Assume Hypothesis 2.3, suppose that $\Theta_{j}, j=1,2$, satisfy the assumptions introduced in Hypothesis 2.4, and denote by $L_{\Theta_{j}, \Omega}$ the operators in (2.21) associated with the sesquilinear forms $\mathfrak{Q}_{\Theta_{j}, \Omega}(\cdot, \cdot), j=1,2$, defined on $H^{1}(\Omega) \times H^{1}(\Omega)$ according to (2.20). Suppose, in addition, that $\Theta_{1}$ satisfies (2.29) and that

$$
\operatorname{Re}\left(\left\langle\gamma_{D} u, \Theta_{2} \gamma_{D} v\right\rangle_{1 / 2}\right) \geqslant\left\langle\gamma_{D}|u|, \Theta_{1} \gamma_{D}|v|\right\rangle_{1 / 2},
$$

for all $u, v \in H^{1}(\Omega)$ such that $u . \bar{v} \geqslant 0$. Then $e^{-t L_{\Theta_{2}, \Omega}}$ is dominated by $e^{-t L_{\Theta_{1}, \Omega}}$, in the sense that

$$
\left|e^{-t L_{\Theta_{2}, \Omega}} f\right| \leqslant e^{-t L_{\Theta_{1}, \Omega}}|f|, \quad f \in L^{2}\left(\Omega ; d^{n} x\right), t \geqslant 0 .
$$

If in addition $\Theta_{1} \geqslant 0$, then

$$
\left|e^{-t L_{\Theta_{2}, \Omega}} f\right| \leqslant e^{-t L_{\Theta_{1}, \Omega}}|f| \leqslant e^{-t L_{N, \Omega}}|f|, \quad f \in L^{2}\left(\Omega ; d^{n} x\right), t \geqslant 0 .
$$

Proof. We have seen at the end of Section 2 that $e^{-t L_{\Theta_{1}, \Omega}}$ is positivity preserving. In addition, the forms $\mathfrak{Q}_{\Theta_{2}, \Omega}$ and $\mathfrak{Q}_{\Theta_{1}, \Omega}$ have the same domain $H^{1}(\Omega)$. We are now in a position to apply Theorem 3.2 . One notes that $u \in H^{1}(\Omega)$ implies $|u| \in H^{1}(\Omega)$, and that

$$
\partial_{k}|u|=\operatorname{Re}\left(\partial_{k} u \cdot \operatorname{sign}(\bar{u})\right), \quad 1 \leqslant k \leqslant n,
$$

where

$$
\operatorname{sign}(\bar{u})(x)= \begin{cases}\frac{\overline{u(x)}}{|u(x)|}, & \text { if } u(x) \neq 0 \\ 0, & \text { if } u(x)=0\end{cases}
$$


Formula (3.10) is well-known (see, e.g., [6, pp. 104-105]). Using (3.10) one concludes that

$$
\begin{aligned}
& \operatorname{Re}\left(\int_{\Omega} d^{n} x\langle\overline{(\nabla u)(x)}, A(x)(\nabla v)(x)\rangle_{\mathbb{C}^{n}}\right) \\
& \quad \geqslant \int_{\Omega} d^{n} x\langle(\nabla|u|)(x), A(x)(\nabla|v|)(x)\rangle_{\mathbb{C}^{n}},
\end{aligned}
$$

for $u, v \in H^{1}(\Omega)$ such that $u \cdot \bar{v} \geqslant 0$. Using (3.12) and assumption (3.7) one infers that assertion $(\mathrm{ii})$ of Theorem 3.2 holds. An application of Theorem 3.2 then yields that (3.8) is satisfied.

Similarly, again by Theorem 3.2 the second inequality in 3.9 holds once we prove that

$$
\mathfrak{Q}_{\Theta_{1}, \Omega}(u, v) \geqslant \mathfrak{Q}_{0, \Omega}(u, v),
$$

for all nonnegative $u, v \in H^{1}(\Omega)$. This inequality follows along the same ideas as above, incorporating the assumption $\Theta_{1} \geqslant 0$.

Remark 3.4. The same proof shows that $e^{-t L_{D, \Omega}}$ is dominated by $e^{-t L_{\Theta_{1}, \Omega}}$ if $\Theta_{1} \geqslant 0$. This domination is also stated explicitly in [4].

Remark 3.5. (i) Under the hypotheses of Theorem 3.3. all semigroups $e^{-t L_{D, \Omega}}$, $e^{-t L_{N, \Omega}}$ and $e^{-t L_{\Theta_{1}, \Omega}}$ for $\Theta_{1} \geqslant 0$ are sub-Markovian and hence extend to contraction semigroups on $L^{\infty}\left(\Omega ; d^{n} x\right)$. In addition, if $\Theta_{2}$ is as in (3.7) then $e^{-t L_{\Theta_{2}, \Omega}}$ extends to a contraction semigroup on $L^{\infty}\left(\Omega ; d^{n} x\right)$. Moreover, all these semigroups extend to strongly continuous semigroups on $L^{p}\left(\Omega ; d^{n} x\right), p \in[1, \infty)$ (holomorphic semigroups for $p \in(1, \infty)$ in appropriate sectors), see $[6$, Chs. 2, 3, 7].

(ii) Condition (2.29) is automatically satisfied in the special case of local Robin boundary conditions considered in Lemma 2.6.

(iii) One can add a potential $0 \leqslant V \in L_{\text {loc }}^{1}\left(\Omega ; d^{n} x\right)$ to all operators in Theorem 3.3 by employing a standard sesquilinear form approach described in [4, Remark 4.8].

Now we turn to the principal new results in this note.

Theorem 3.6. Let $\Omega$ be a connected bounded Lipschitz domain in $\mathbb{R}^{n}$. Assume Hypothesis 2.3, suppose that $\Theta$ satisfies the assumptions introduced in Hypothesis 2.4, and that

$$
\operatorname{Re}\left(\left\langle\gamma_{D} u, \Theta \gamma_{D} v\right\rangle_{1 / 2}\right) \geqslant 0
$$

for $u, v \in H^{1}(\Omega)$ with $u . \bar{v} \geqslant 0$. Then there exist finite constants $C>0, c>0$ such that (for $t>0$, a.e. $x, y \in \Omega$ )

$$
\left|K_{\Theta, \Omega}(t, x, y)\right| \leqslant C \max \left(t^{-n / 2}, 1\right) \exp \left[-c|x-y|^{2} / t\right] .
$$

In addition, assuming

$$
\left\langle\gamma_{D} 1, \Theta \gamma_{D} 1\right\rangle_{1 / 2} \neq 0
$$

where 1 denotes the constant function with value 1 on $\Omega$, then

$$
\lambda_{1, \Theta, \Omega}=\inf \sigma\left(L_{\Theta, \Omega}\right)>0,
$$

and there exist finite constants $C>0, c>0$, such that the Robin heat kernel $K_{\Theta, \Omega}(t, \cdot, \cdot)$ satisfies (for $t>0$, a.e. $x, y \in \Omega$ ),

$$
\left|K_{\Theta, \Omega}(t, x, y)\right| \leqslant C t^{-n / 2}(1+t)^{n / 2} e^{-\lambda_{1, \Theta, \Omega} t} \exp \left[-c|x-y|^{2} / t\right] .
$$


Proof. On one hand, Theorem 3.3 and observation (2.6) imply the following comparison for the Robin and Neumann heat kernels (for $t>0$, a.e. $x, y \in \Omega$ )

$$
\left|K_{\Theta, \Omega}(t, x, y)\right| \leqslant K_{N, \Omega}(t, x, y) \text {. }
$$

On the other hand, it is known that on a bounded Lipschitz domain $\Omega$, the Neumann heat kernel $K_{N, \Omega}(t, x, y)$ enjoys the Gaussian upper bound (for $t>0$, a.e. $x, y \in \Omega$ )

$$
K_{N, \Omega}(t, x, y) \leqslant C \max \left(t^{-n / 2}, 1\right) \exp \left[-c|x-y|^{2} / t\right],
$$

where $C$ and $c$ are positive finite constants. Combining (3.20) with (3.19), one obtains (3.15). By (2.23) (i.e., the compact embedding of $H^{1}(\Omega)$ into $L^{2}(\Omega)$ ), $L_{\Theta, \Omega}$ has purely discrete spectrum. Let $\lambda_{1, \Theta, \Omega}:=\inf \sigma\left(L_{\Theta, \Omega}\right)$. Then $\lambda_{1, \Theta, \Omega}$ is the smallest eigenvalue of $L_{\Theta, \Omega}$ and we claim that $\lambda_{1, \Theta, \Omega}$ is strictly positive. To justify this claim, we reason by contradiction and note that if $\lambda_{1, \Theta, \Omega}=0$ then hypothesis (3.14) (with $u=v$ ) and the fact that $L_{\Theta, \Omega} \geqslant 0$ (cf. (2.20) ) would imply the existence of a nonzero $u \in \operatorname{dom}\left(L_{\Theta, \Omega}\right)$ such that

$$
\left(u, L_{\Theta, \Omega} u\right)_{L^{2}\left(\Omega ; d^{n} x\right)}+\left\langle\gamma_{D} u, \Theta \gamma_{D} u\right\rangle_{1 / 2}=0 .
$$

Since $\left\langle\gamma_{D} u, \Theta \gamma_{D} u\right\rangle_{1 / 2} \geqslant 0$ by (3.14), one concludes that $\left(u, L_{\Theta, \Omega} u\right)_{L^{2}\left(\Omega ; d^{n} x\right)}=0$ and $\left\langle\gamma_{D} u, \Theta \gamma_{D} u\right\rangle_{1 / 2}=0$. The first equality together with the assumed ellipticity of $L$ implies that $u$ is constant on $\Omega$. The second equality together with (3.16) then yields the desired contradiction. This proves (3.17). Next, we improve on (3.15) to obtain (3.18). Obviously, we may consider $t \geqslant 1$, only. By [6, Lemma 6.5] and (3.15) we obtain (for $t>0$, a.e. $x, y \in \Omega$ )

$$
\left|K_{\Theta, \Omega}(t, x, y)\right| \leqslant C t^{-n / 2} e^{-\lambda_{1, \Theta, \Omega} t}\left[1+\lambda_{1, \Theta, \Omega} t\right]^{n / 2} .
$$

Now since $\Omega$ is bounded it has finite diameter. Therefore, $|x-y| \leqslant \operatorname{diam}(\Omega)$ for all $x, y \in \Omega$. From (3.22) we may estimate that (for $t \geqslant 1$, a.e. $x, y \in \Omega$ )

$$
\begin{aligned}
\left|K_{\Theta, \Omega}(t, x, y)\right| & \leqslant C t^{-n / 2} e^{-\lambda_{1, \Theta, \Omega} t}\left[1+\lambda_{1, \Theta, \Omega} t\right]^{n / 2} \\
& =C t^{-n / 2} e^{-\lambda_{1, \Theta, \Omega} t} e^{-c|x-y|^{2} / t} e^{c|x-y|^{2} / t}\left[1+\lambda_{1, \Theta, \Omega} t\right]^{n / 2} \\
& \leqslant C t^{-n / 2} e^{-\lambda_{1, \Theta, \Omega} t} e^{-c|x-y|^{2} / t} e^{c[\operatorname{diam}(\Omega)]^{2}}\left[1+\lambda_{1, \Theta, \Omega} t\right]^{n / 2},
\end{aligned}
$$

completing the proof.

Remark 3.7. While (3.15) extends the principal result obtained in [4] (cf. Theorem 3.1), it still produces a Neumann-type bound (see also (3.6)) for $K_{\Theta, \Omega}(t, x, y)$, $t>0$, a.e. $x, y \in \Omega$. It is the additional condition (3.16) that permits one to derive the considerably improved bound (3.18) containing the exponential decay factor $e^{-\lambda_{1, \Theta, \Omega} t}, t>0$.

Remark 3.8. (i) From Theorem 3.6 one obtains the following estimates for the Robin Green function. For $\lambda>0$, and a.e. $x, y \in \Omega$,

$$
\left|G_{\Theta, \Omega}(\lambda, x, y)\right| \leqslant\left\{\begin{array}{ll}
C_{a_{0}, a_{1}, \lambda, \Omega, n}|x-y|^{2-n}, & n \geqslant 3, \\
C_{a_{0}, a_{1}, \lambda, \Omega}\left|\ln \left(1+|x-y|^{-1}\right)\right|, & n=2,
\end{array} \quad x \neq y .\right.
$$

This follows as usual by writing the resolvent as the Laplace transform of the semigroup and hence the Green function at $\lambda$ as the Laplace transform of the heat kernel (cf. 4, App. C]).

(ii) The semigroup $e^{-t L_{\Theta, \Omega}}$ is bounded holomorphic on $L^{p}\left(\Omega ; d^{n} x\right)$ in the sector $\{z \in \mathbb{C}|| \arg (z) \mid<\pi / 2\}$ for all $p \in[1, \infty)$. In particular, the generator 
of the corresponding semigroup has (minus) spectrum contained in $[0, \infty)$ and is $p$-independent. See [6, Ch. 7].

(iii) The operator $L_{\Theta, \Omega}$ has a bounded holomorphic functional calculus and one even has a spectral multiplier result, see, 6, Theorem 7.23].

Define the metric $\rho(\cdot, \cdot)$ on $\Omega$ by setting, for each $x, y \in \Omega$,

$$
\begin{array}{r}
\rho(x, y):=\sup \left\{\varphi(x)-\varphi(y) \mid \varphi \in W^{1, \infty}\left(\mathbb{R}^{n}\right),\right. \text { real-valued, and } \\
\left.\sum_{j, k=1}^{n} a_{j, k}(\xi) \partial_{j} \varphi(\xi) \partial_{k} \varphi(\xi) \leqslant 1 \text { for a.e. } \xi \in \Omega\right\} .
\end{array}
$$

This is the metric associated with the coefficients $a_{j, k}$. By ellipticity, $\rho(\cdot, \cdot)$ is clearly (two-sided, pointwise) comparable with the standard Euclidean metric. Following the method in [7] for Schrödinger-type operators one can actually derive a sharper estimate using $\rho(\cdot, \cdot)$ instead of the Euclidean metric.

Proposition 3.9. Suppose that the assumptions of Theorem 3.6 are satisfied. In addition, assume that

$$
\left\langle\gamma_{D}\left(e^{\varphi} u\right), \Theta \gamma_{D}\left(e^{-\varphi} u\right)\right\rangle_{1 / 2} \geqslant\left\langle\gamma_{D} u, \Theta \gamma_{D} u\right\rangle_{1 / 2}
$$

for all real-valued $\varphi \in C^{\infty}\left(\mathbb{R}^{n}\right) \cap L^{\infty}\left(\mathbb{R}^{n}\right)$. Then there exist finite constants $C>0$, $c>0$ such that the Robin heat kernel $K_{\Theta, \Omega}(t, \cdot, \cdot)$ satisfies $($ for $t>0$, a.e. $x, y \in \Omega$ ),

$$
\begin{aligned}
\left|K_{\Theta, \Omega}(t, x, y)\right| \leqslant & C^{\prime} t^{-n / 2} e^{-\lambda_{1, \Theta, \Omega} t}\left[1+\lambda_{1, \Theta, \Omega} t+\left[\rho(x, y)^{2} / t\right]^{n / 2}\right. \\
& \times \exp \left[-\rho(x, y)^{2} /(4 t)\right] .
\end{aligned}
$$

Proof. In order to prove estimate (3.27), assuming condition (3.26), we fix $\lambda \in \mathbb{R}$ and a real-valued function $\varphi \in W^{1, \infty}\left(\mathbb{R}^{n}\right)$ such that

$$
\sum_{j, k=1}^{n} a_{j, k}(x) \partial_{j} \varphi(x) \partial_{k} \varphi(x) \leqslant 1 \text { for a.e. } x \in \Omega .
$$

Following Davies' perturbation method (see, e.g., [2, Ch. 3]), introduce $S_{\lambda}(t):=$ $e^{\lambda \varphi} e^{-t L_{\Theta, \Omega}} e^{-\lambda \varphi}$. This semigroup has an integral kernel given by

$$
e^{\lambda(\varphi(x)-\varphi(y))} K_{\Theta, \Omega}(t, x, y) \text { a.e. } x, y \in \Omega, t>0 .
$$

Using Theorem 3.6, one obtains for any $\delta \in(0,1)$ there exist finite constants $c_{\delta}, C_{\delta}>0$ such that (for $t>0$, a.e. $x, y \in \Omega$ ),

$$
e^{\lambda[\varphi(x)-\varphi(y)]}\left|K_{\Theta, \Omega}(t, x, y)\right| \leqslant e^{\frac{1}{\delta} \lambda^{2} t} e^{\delta[\varphi(x)-\varphi(y)]^{2} / t} C_{\delta} t^{-n / 2} \exp \left[-c_{\delta}|x-y|^{2} / t\right] \text {. }
$$

Choosing $\delta>0$ sufficiently small and using (3.28) one obtains that, on the one hand (for $t>0$, a.e. $x, y \in \Omega$ ),

$$
e^{\lambda[\varphi(x)-\varphi(y)]}\left|K_{\Theta, \Omega}(t, x, y)\right| \leqslant e^{\frac{1}{\delta} \lambda^{2} t} C t^{-n / 2} .
$$

On the other hand, the semigroup $S_{\lambda}(t)$ is associated with the sesquilinear form

$$
\mathfrak{Q}_{\Theta, \Omega}\left(e^{-\lambda \varphi} u, e^{\lambda \varphi} v\right), \quad u, v \in H^{1}(\Omega),
$$

where $\mathfrak{Q}_{\Theta, \Omega}$ is the form of $L_{\Theta, \Omega}$. One verifies that

$$
\mathfrak{Q}_{\Theta, \Omega}\left(e^{-\lambda \varphi} u, e^{\lambda \varphi} u\right)=\sum_{j, k=1}^{n} \int_{\Omega} d^{n} x a_{j, k} \overline{\partial_{j} u} \partial_{k} u-\lambda^{2} \sum_{j, k=1}^{n} \int_{\Omega} d^{n} x a_{j, k} \partial_{j} \varphi \partial_{k} \varphi|u|^{2}
$$




$$
+\left\langle\gamma_{D}\left(e^{-\lambda \varphi} u\right), \Theta \gamma_{D}\left(e^{-\varphi} u\right)\right\rangle_{1 / 2}, \quad u \in H^{1}(\Omega) .
$$

Thus, using (3.28) and (3.26),

$\operatorname{Re}\left(\mathfrak{Q}_{\Theta, \Omega}\left(e^{-\lambda \varphi} u, e^{\lambda \varphi} u\right)\right) \geqslant \mathfrak{Q}_{\Theta, \Omega}(u, u)-\lambda^{2} \int_{\Omega} d^{n} x|u|^{2} \geqslant\left(\lambda_{1, \Theta, \Omega}-\lambda^{2}\right) \int_{\Omega} d^{n} x|u|^{2}$, $u \in H^{1}(\Omega)$.

The latter inequality implies the following estimate

$$
\left\|S_{\lambda}(t)\right\|_{\mathcal{B}\left(L^{2}\left(\Omega ; d^{n} x\right)\right)} \leqslant e^{-\left(\lambda_{1, \Theta, \Omega}-\lambda^{2}\right) t} .
$$

Now we proceed as in the beginning of the poof of Theorem 3.6. Estimates (3.31), (3.35), and [6, Lemma 6.5] imply (for $t>0$, a.e. $x, y \in \Omega$ ),

$$
e^{\lambda[\varphi(x)-\varphi(y)]}\left|K_{\Theta, \Omega}(t, x, y)\right| \leqslant C t^{-n / 2} e^{-\lambda_{1, \Theta, \Omega} t} e^{\lambda^{2} t}\left[1+\lambda_{1, \Theta, \Omega} t+\delta^{\prime} \lambda^{2} t\right]^{n / 2}
$$

with $\delta^{\prime}:=\delta^{-1}-1$. We arrive at (for $t>0$, a.e. $x, y \in \Omega$ ),

$$
\left|K_{\Theta, \Omega}(t, x, y)\right| \leqslant C^{\prime} t^{-n / 2} e^{-\lambda_{1, \Theta, \Omega} t} e^{\lambda^{2} t}\left[1+\lambda_{1, \Theta, \Omega} t+\lambda^{2} t\right]^{n / 2} e^{-\lambda[\varphi(x)-\varphi(y)]} .
$$

Choosing $\lambda=[\varphi(x)-\varphi(y)] /(2 t)$ and optimizing over $\varphi$ yields (3.27).

One notes that while condition (3.26) may not be automatically satisfied in the presence of nonlocal Robin boundary conditions, it is certainly fulfilled in the case of local Robin boundary conditions.

\section{Some illustrations}

We conclude this note with a number of concrete examples illustrating Theorem 3.6.

Assuming throughout this section that $n \geqslant 3$, one recalls that

$$
E_{n}(x):=\frac{1}{(n-2) \omega_{n-1}} \frac{1}{|x|^{n-2}}, \quad x \in \mathbb{R}^{n} \backslash\{0\},
$$

is the fundamental solution for (minus) the Laplacian $-\Delta$ in $\mathbb{R}^{n}$. Here $\omega_{n-1}=$ $2 \pi^{n / 2} / \Gamma(n / 2)(\Gamma(\cdot)$ the gamma function, cf. [1, Sect. 6.1]) represents the area of the unit sphere $S^{n-1}$ in $\mathbb{R}^{n}$.

Next, suppose that $\Omega \subseteq \mathbb{R}^{n}$ is a Lipschitz domain with compact boundary, and denote by $\omega$ the canonical surface measure on $\partial \Omega$. Then the (boundary-to-boundary version of the) harmonic single layer associated with $\Omega$ is the integral operator of formal convolution with $E_{n}$, that is,

$$
(S f)(\xi):=-\int_{\partial \Omega} d^{n-1} \omega(\eta) E_{n}(\xi-\eta) f(\eta), \quad \xi \in \partial \Omega .
$$

One observes that in the special case where $\partial \Omega \in C^{\infty}$, it follows that $S$ is a classical pseudodifferential operator of order -1 . This description of $S$ is tightly connected with the strong regularity assumption on the boundary of $\Omega$, and fails to materialize in the presence of just one boundary irregularity. Nonetheless, $S$ continues to enjoy remarkable properties even when considered on rough surfaces, as in the presently assumed Lipschitz setting. Some of its basic properties relevant for us here are as follows:

$$
S: L^{2}\left(\partial \Omega ; d^{n-1} \omega\right) \longrightarrow L^{2}\left(\partial \Omega ; d^{n-1} \omega\right) \text { is linear, compact, }
$$
nonnegative, injective, with range $H^{1}(\partial \Omega)$. 
Functional calculus then yields that

$$
S^{i \gamma} \text { is a unitary operator on } L^{2}\left(\partial \Omega ; d^{n-1} \omega\right) \text { for each } \gamma \in \mathbb{R} \text {. }
$$

Also, starting from the fact that

$$
S: L^{2}\left(\partial \Omega ; d^{n-1} \omega\right) \longrightarrow H^{1}(\partial \Omega) \text { is a linear, bounded, isomorphism, }
$$

by duality and interpolation we obtain that

$$
S: H^{s-1}(\partial \Omega) \longrightarrow H^{s}(\partial \Omega) \text { is a linear, bounded, isomorphism. }
$$

Functional calculus may be also used to define complex and fractional powers of $S$.

Lemma 4.1. For each $\alpha \in[0,1]$ and $s \in[0, \alpha]$,

$$
S^{-\alpha}: H^{s}(\partial \Omega) \longrightarrow H^{s-\alpha}(\partial \Omega) \text { is a linear, bounded, isomorphism. }
$$

Proof. In a first stage, we shall show that

$$
S^{\alpha}: L^{2}\left(\partial \Omega ; d^{n-1} \omega\right) \longrightarrow H^{\alpha}(\partial \Omega) \text { is a linear, bounded, }
$$

$$
\text { isomorphism, for each } \alpha \in[0,1] \text {. }
$$

To justify this, note that the family of operators $\left\{S^{z}\right\}_{z \in \mathbb{S}}$ (with $\mathbb{S}=\{z \in \mathbb{C} \mid 0 \leqslant$ $\operatorname{Re}(z) \leqslant 1\}$ denoting a closed complex strip), depends analytically on the parameter $z$ in the interior of $\mathbb{S}$, when viewed as a mapping with values in $\mathcal{B}\left(L^{2}\left(\partial \Omega ; d^{n-1} \omega\right)\right)$, and

$$
\begin{aligned}
& S^{z}: L^{2}\left(\partial \Omega ; d^{n-1} \omega\right) \longrightarrow H^{\operatorname{Re}(z)}(\partial \Omega) \text { is a linear, bounded, } \\
& \text { isomorphism when } \operatorname{Re}(z)=0 \text { or } \operatorname{Re}(z)=1,
\end{aligned}
$$

due to (4.4) and (4.5). Then (4.8) follows from this and Stein's complex interpolation theorem for analytic families of operators. Having established (4.8), via duality and interpolation, one then obtains that

$$
\begin{gathered}
S^{\alpha}: H^{s-\alpha}(\partial \Omega) \longrightarrow H^{s}(\partial \Omega) \text { is a linear, bounded, } \\
\text { isomorphism whenever } \alpha \in[0,1] \text { and } s \in[0, \alpha] .
\end{gathered}
$$

Taking inverses, this finally yields (4.7).

The next step is to prove a nondegeneracy condition for fractional powers of the harmonic single layer, in the spirit of (3.16).

Lemma 4.2. If 1 denotes the constant function 1 in $\Omega$, then for each $\alpha \in[0,1]$ one has

$$
\left\langle\gamma_{D} 1, S^{-\alpha} \gamma_{D} 1\right\rangle_{1 / 2}>0
$$

Proof. Since $\gamma_{D} 1 \in H^{s}(\partial \Omega)$ for each $s \in[0,1]$, from (4.7) (used with $s=\alpha$ ) one deduces that

$$
S^{-\alpha} \gamma_{D} 1 \in L^{2}\left(\partial \Omega ; d^{n-1} \omega\right) \text { for each } \alpha \in[0,1] .
$$

Next, fix $\alpha \in[0,1]$. Then on account of the self-adjointness of $S$ and (4.12) one can write

$$
\left\langle\gamma_{D} 1, S^{-\alpha} \gamma_{D} 1\right\rangle_{1 / 2}=\left\langle S^{-\alpha / 2} \gamma_{D} 1, S^{-\alpha / 2} \gamma_{D} 1\right\rangle_{1 / 2}=\int_{\partial \Omega} d^{n-1} \omega\left|S^{-\alpha / 2} \gamma_{D} 1\right|^{2}
$$

Given that Lemma 4.1 ensures that the function $S^{-\alpha / 2} \gamma_{D} 1$ is not identically zero on $\partial \Omega$, the claim in (4.11) now readily follows from (4.13). 
After this preamble, we are in a position to prove the following result which identifies a class of highly nonlocal operators satisfying Hypothesis 2.4 along with a nondegeneracy condition in the spirit of (3.16). We recall that $M_{\theta}$ stands for the operator of pointwise multiplication by the measurable function $\theta$.

Theorem 4.3. Assume $\theta: \partial \Omega \rightarrow[0, \infty)$ is a function that is strictly positive on a subset of $\partial \Omega$ of positive $d^{n-1} \omega$ measure and satisfies $\theta \in L^{p}\left(\partial \Omega ; d^{n-1} \omega\right)$, where

$$
p=n-1 \text { if } n>2 \text {, and } p \in(1, \infty] \text { if } n=2 \text {. }
$$

Then for any given number $\delta>0$ there exists $\varepsilon>0$ with the property that for each $\alpha \in[1 / 2,1)$ the operator

$$
\Theta:=c_{1} M_{\theta}+c_{2} S^{-\alpha}+c_{3} \varepsilon S^{-1}, \quad c_{j} \geqslant 0,1 \leqslant j \leqslant 3, \quad c_{1} c_{2} c_{3} \neq 0,
$$

satisfies the conditions stipulated in Hypothesis 2.4 for the given $\delta$, as well as the nondegeneracy condition

$$
\left\langle\gamma_{D} 1, \Theta \gamma_{D} 1\right\rangle_{1 / 2} \neq 0
$$

Proof. Decompose $\Theta=\Theta^{(1)}+\Theta^{(2)}+\Theta^{(3)}$, where

$$
\Theta^{(1)}:=c_{1} M_{\theta}, \quad \Theta^{(2)}:=c_{2} S^{-\alpha}, \quad \Theta^{(3)}:=c_{3} \varepsilon S^{-1} .
$$

From Lemma 2.6 and the fact that $\theta$ is positive on a subset of $\partial \Omega$ of positive $d^{n-1} \omega$ measure, it follows that

$$
\begin{aligned}
& \Theta^{(1)} \in \mathcal{B}\left(H^{1 / 2}(\partial \Omega), H^{-1 / 2}(\partial \Omega)\right), \\
& \Theta^{(1)} \text { is self-adjoint in this context and }\left\langle\gamma_{D} 1, \Theta^{(1)} \gamma_{D} 1\right\rangle_{1 / 2}>0 \text { if } c_{1}>0 .
\end{aligned}
$$

Next, since $S^{-\alpha}$ maps $H^{1 / 2}(\partial \Omega)$ boundedly into $H^{1 / 2-\alpha}(\partial \Omega)$ by Lemma 4.1 (used here with $s=1 / 2$ and $\alpha \in[1 / 2,1)$ ), and since $H^{1 / 2-\alpha}(\partial \Omega)$ embeds compactly into $H^{1 / 2}(\partial \Omega)$ given that we are currently assuming $\alpha<1$, it follows that

$$
\Theta^{(2)} \in \mathcal{B}_{\infty}\left(H^{1 / 2}(\partial \Omega), H^{-1 / 2}(\partial \Omega)\right) .
$$

Moreover, it is clear from the self-adjointness of $S$ that $\Theta^{(2)}$ is self-adjoint in the context of $\mathcal{B}\left(H^{1 / 2}(\partial \Omega), H^{-1 / 2}(\partial \Omega)\right)$. In addition, Lemma 4.2 yields

$$
\left\langle\gamma_{D} 1, \Theta^{(2)} \gamma_{D} 1\right\rangle_{1 / 2}>0 \text { if } c_{2}>0 .
$$

Similar considerations, based on Lemma 4.1 (used with $\alpha=1$ and $s=1 / 2$ ) and Lemma 4.2 (used with $\alpha=1$ ), prove that

$$
\begin{aligned}
& \Theta^{(3)} \in \mathcal{B}\left(H^{1 / 2}(\partial \Omega), H^{-1 / 2}(\partial \Omega)\right), \\
& \Theta^{(3)} \text { is self-adjoint in this context and }\left\langle\gamma_{D} 1, \Theta^{(3)} \gamma_{D} 1\right\rangle_{1 / 2}>0 \text { if } c_{3}>0 .
\end{aligned}
$$

Finally, it remains to observe that, given any $\delta>0$, Lemma 4.1 may be invoked (with $\alpha=1$ and $s=1 / 2$ ) in order to find a number $\varepsilon>0$ small enough so that

$$
\left\|\Theta^{(3)}\right\|_{\mathcal{B}\left(H^{1 / 2}(\partial \Omega), H^{-1 / 2}(\partial \Omega)\right)}<\delta .
$$

The above analysis then proves that $\Theta$ from (4.15) satisfies all conditions in Hypothesis 2.4 as well as the nondegeneracy condition (4.16). 
Another, more elementary, example of a nonlocal operator satisfying Hypothesis 2.4 as well as the nondegeneracy condition (3.16) may be produced as follows. We retain the assumption that $\Omega \subseteq \mathbb{R}^{n}$ is a Lipschitz domain with compact boundary and consider a measurable kernel

$$
k: \partial \Omega \times \partial \Omega \rightarrow \mathbb{C}
$$

satisfying the symmetry condition

$$
k(\xi, \eta)=\overline{k(\eta, \xi)} \quad \xi, \eta \in \partial \Omega,
$$

and such that

$$
\begin{aligned}
& \int_{\partial \Omega} \int_{\partial \Omega} d^{n-1} \omega(\xi) d^{n-1} \omega(\eta)|k(\xi, \eta)|<+\infty \\
& \int_{\partial \Omega} d^{n-1} \omega(\eta)\left|\int_{\partial \Omega} d^{n-1} \omega(\xi) k(\xi, \eta)\right|^{2} \neq 0 .
\end{aligned}
$$

Moreover, suppose that the kernel $k$ is sufficiently decent such that the integral operator

$$
(A f)(\xi):=\int_{\partial \Omega} d^{n-1} \omega(\eta) k(\xi, \eta) f(\eta), \quad \xi \in \partial \Omega
$$

satisfies

$$
A \in \mathcal{B}_{\infty}\left(H^{1 / 2}(\partial \Omega), L^{2}\left(\partial \Omega ; d^{n-1} \omega\right)\right) .
$$

For example, condition (4.28) holds if $A$ is compact on $L^{2}\left(\partial \Omega ; d^{n-1} \omega\right)$, which is always the case if $k$ satisfies the stronger Hilbert-Schmidt condition

$$
\int_{\partial \Omega} \int_{\partial \Omega} d^{n-1} \omega(\xi) d^{n-1} \omega(\eta)|k(\xi, \eta)|^{2}<+\infty
$$

in place of condition (4.25). Assuming that (4.27) holds, one then deduces that

$$
A^{*} \in \mathcal{B}_{\infty}\left(L^{2}\left(\partial \Omega ; d^{n-1} \omega\right), H^{-1 / 2}(\partial \Omega)\right)
$$

and, ultimately, that

$$
\Theta:=A^{*} A \in \mathcal{B}_{\infty}\left(H^{1 / 2}(\partial \Omega), H^{-1 / 2}(\partial \Omega)\right) .
$$

Moreover, the linear operator $\Theta$ defined in (4.31) is self-adjoint in the context of $\mathcal{B}\left(H^{1 / 2}(\partial \Omega), H^{-1 / 2}(\partial \Omega)\right)$ and

$$
\left\langle\gamma_{D} 1, \Theta \gamma_{D} 1\right\rangle_{1 / 2}=\int_{\partial \Omega} d^{n-1} \omega\left|A \gamma_{D} 1\right|^{2}=\int_{\partial \Omega} d^{n-1} \omega(\eta)\left|\int_{\partial \Omega} d^{n-1} \omega(\xi) k(\xi, \eta)\right|^{2} \neq 0
$$

by condition (4.26). The bottom line is that that the operator $\Theta$ in (4.31) satisfies Hypothesis 2.4 (taking $\Theta^{(1)}=\Theta^{(3)}=0$ ), as well as the nondegeneracy condition (4.16).

\section{REFERENCES}

[1] M. Abramowitz and I. A. Stegun, Handbook of Mathematical Functions, Dover, New York, 1972 .

[2] E. B. Davies, Heat kernels and spectral theory, Cambridge Tracts in Mathematics, vol. 92, Cambridge University Press, Cambridge, 1989. MR990239 (90e:35123)

[3] F. Gesztesy and M. Mitrea, Nonlocal Robin Laplacians and some remarks on a paper by Filonov on eigenvalue inequalities, J. Differential Equations 247 (2009), no. 10, 2871-2896, DOI 10.1016/j.jde.2009.07.007. MR2568160(2010k:35079) 
[4] F. Gesztesy, M. Mitrea, and R. Nichols, Heat kernel bounds for elliptic partial differential operators in divergence form with Robin-type boundary conditions, J. Analyse Math. (to appear).

[5] E.-M. Ouhabaz, Invariance of closed convex sets and domination criteria for semigroups, Potential Anal. 5 (1996), no. 6, 611-625, DOI 10.1007/BF00275797. MR.1437587 (98a:47041)

[6] E.-M. Ouhabaz, Analysis of heat equations on domains, London Mathematical Society Monographs Series, vol. 31, Princeton University Press, Princeton, NJ, 2005. MR2124040 (2005m:35001)

[7] E.-M. Ouhabaz, Sharp Gaussian bounds and $L^{p}$-growth of semigroups associated with elliptic and Schrödinger operators, Proc. Amer. Math. Soc. 134 (2006), no. 12, 3567-3575 (electronic), DOI 10.1090/S0002-9939-06-08430-9. MR2240669 (2007g:35029)

Department of Mathematics, University of Missouri, Columbia, Missouri 65211

E-mail address: gesztesyf@missouri.edu

$U R L:$ http://www.math.missouri.edu/personnel/faculty/gesztesyf .html

Department of Mathematics, University of Missouri, Columbia, Missouri 65211

E-mail address: mitream@missouri.edu

URL: http://www.math.missouri.edu/personnel/faculty/mitream.html

Mathematics Department, The University of Tennessee at Chattanooga, 415 EMCS

Building, Dept. 6956, 615 McCallie Ave, Chattanooga, Tennessee 37403

E-mail address: roger-nichols@utc.edu

URL: http://www.utc.edu/faculty/roger-nichols/index.php

University of Bordeaux, Institut de Mathématiques (IMB), Equipe d'Analyse, 351, Cours de la Libération, 33405 Talence, France

E-mail address: elmaati.ouhabaz@math.u-bordeaux1.fr

URL: http: //www.math.u-bordeaux1.fr/ eouhabaz/ 